\title{
Coordination of dual-channel supply chain with perfect product considering sales effort
}

\author{
Hu, H..$^{a}{ }^{,}$, Wu, Q. ${ }^{a}$, Han, S. ${ }^{a}$, Zhang, Z. ${ }^{a}$ \\ ${ }^{a}$ School of Economics and Management, Yanshan University, Qinhuangdao, P.R. China
}

\begin{abstract}
A B S T R A C T
As more and more people use e-commerce for shopping, manufacturers are willing to open online sales channels in order to obtain more profits. This paper discusses a dual-channel supply chain (DCSC) composed of a retailer with a traditional channel and a manufacturer with a direct channel. In the external environment of uncertain market demand and defective products produced by manufacturers, manufacturers make efforts to promote online products, and consumers have free rider behaviour. Therefore, three game models under the leadership of manufacturers are established: (a) noncooperative game model; (b) coordination model under revenue-sharing contract; (c) coordination model under profit-sharing contract. The results indicate that the product defect rate has a certain influence on channel pricing and sale efforts. The competition between the actors of the dual-channel is beneficial to the consumers who pursue the price. Considering the overall profit of the DCSC, the cooperation between the manufacturer and retailer is more profitable than the channel competition, and they are more willing to make product sale efforts. The retailer's expected profit under revenuesharing contract is less than that under profit-sharing contract, but the total profit of coordination model is more than the latter.
\end{abstract}

ARTICLE INFO

Keywords:

e-commerce;

Supply chain;

Dual-channel supply chain (DCSC);

Defective product;

Manufacturer sales effort;

Coordination;

Game theory

*Corresponding author:

huhaiju@ysu.edu.cn

(Hu, H.)

Article history:

Received 22 April 2020

Revised 20 June 2020

Accepted 25 June 2020

(C) 2020 CPE, University of Maribor. All rights reserved.

\section{References}

[1] Milić, B., Rosi, B., Gumzej, R. (2019). An approach to E-marketplace automation, Tehnički Vjesnik - Technical Gazette, Vol.26, No. 3, 639-649, doi: 10.17559/tv-20171201150248.

[2] Burinskiene, A. (2018). New challenges for supply chain: Electronic invoicing and its use perspective, Journal of Logistics, Informatics and Service Science, Vol. 5, No. 1, 31-42.

[3] Chen, J., Liang, L., Yao, D.-Q., Sun, S. (2017). Price and quality decisions in dual-channel supply chains, European Journal of Operational Research, Vol. 259, No. 3, 935-948, doi: 10.1016/i.ejor.2016.11.016.

[4] Taleizadeh, A.A., Khanbaglo, M.P.S., Cárdenas-Barrón, L.E. (2016). An EOQ inventory model with partial backordering and reparation of imperfect products, International Journal of Production Economics, Vol. 182, 418-434, doi: 10.1016/i.ijpe.2016.09.013.

[5] Modak, N.M., Panda, S., Sana, S.S. (2016). Three-echelon supply chai coordination considering duo polistic retailers with perfect quality products, International Journal of Production Economics, Vol. 182, 564-578, doi: 10.1016/i.ijpe.2015.05.021.

[6] Rad, M.A., Khoshalhan, F., Glock, C.H. (2018). Optimal production and distribution policies for a two-stage supply chain with imperfect items and pric e-and advertisement-sensitive demand: A note, Applied Mathematical Modelling, Vol. 57, 625-632, doi: 10.1016/i.apm.2016.11.003.

[7] Li, W., Chen, J., Liang, G., Chen, B. (2018). Money -back guarantee and personalized pricing in a Stackelberg manufacturer's dual-channel supply chain, International Journal of Production Economics, Vol. 197, 84-98, doi: 10.1016 Li.ijpe.2017.12.027. 
[8] Hu, H., Wu, Q., Zhang, Z., Han, S. (2019). Effect of the manufacturer quality inspection policy on the supply chain decision-making and profits, Advances in Production Engineering \& Management, Vol. 14, No. 4, 472-482, doi: 10.14743/apem2019.4.342.

[9] Tsao, Y.-C. (2015). Cooperative promotion under demand uncertainty, International Journal of Production Economics, Vol. 167, 45-49, doi: 10.1016/i.ijpe.2015.05.023.

[10] De Giovanni, P. (2011). Quality improvement vs. advertising support: Which strategy works better for a manufacturer?, European Journal of Operational Research, Vol. 208, No. 2, 119-130, doi: 10.1016/i.ejor.2010.08.003.

[11] Pu, X., Gong, L., Han, X. (2017). Cons umer free riding: Coordinating sales effort in a dual-channel supply chain, Electronic Commerce Research and Applications, Vol. 22, 1-12, doi: 10.1016/i.elerap.2016.11.002.

[12] Li, B., Hou, P.-W., Li, Q.-H. (2015). Cooperative advertising in a dual-channel supply chain with a fairness concern of the manufacturer, IMA Journal of Management Mathematics, Vol. 28, No. 2, 259-277, doi: 10.1093/imaman/ dpv025.

[13] Chen, X., Zhang, W., Gu, W. (2019). Coordination of two-echelon pharmaceutical supply chain with dual-channel considering promotion behavior and consumer preference, Industrial Engineering \& Management, Vol. 24, No. 139, 24-33.

[14] Ranjan, A., Jha, J.K. (2019). Pricing and coordination strategies of a dual-channel supply chain considering green quality and sales effort, Journal of Cleaner Production, Vol. 218, 409-424, doi: 10.1016/i.jclepro.2019.01.297.

[15] Wang, L., Song, Q. (2020). Pricing policies for dual-channel supply chain with green investment and sales effort under uncertain demand, Mathematics and Computers in Simulation, Vol. 171, 79-93, doi: 10.1016/i.matcom. 2019.08.010.

[16] Nazifa, T.H., Ramachandran, K.K. (2019). Information sharing in supply chain management: A case study between the cooperative partners in manufacturing indus try, Journal of System and Management Sciences, Vol. 9, No. 1, 19-47.

[17] Zhu, X.D., Li, B.Y., Wang, Z. (2017). A study on the manufacturing decision-making and optimization of hybridchannel supply chain for original equipment manufacturer, Advances in Production Engineering \& Management, Vol. 12, No. 2, 185-195, doi: 10.14743/apem2017.2.250.

[18] Cai, G., Zhang, Z.G., Zhang, M. (2009). Game theoretical perspectives on dual-channel supply chain competition with price discounts and pricing schemes, International Journal of Production Economics, Vol. 117, No. 1, 80-96, doi: 10.1016/i.ijpe.2008.08.053.

[19] Chen, J., Zhang, H., Sun, Y. (2012). Implementing coordination contracts in a manufacturer Stackelberg dualchannel supply chain, Omega, Vol. 40, No. 5, 571-583, doi: 10.1016/i.omega.2011.11.005.

[20] He, L., Zhang, X., Wang, Q.P., Hu, C.L. (2018). Game theoretic analysis of supply chain bas ed on mean-variance approach under cap-and-trade policy, Advances in Production Engineering \& Management, Vol. 13, No. 3, 333344, doi: 10.14743/apem2018.3.294.

[21] Liu, H., Sun, S., Lei, M., Leong, G.K., Deng, H. (2016). Research on cost information sharing and channel choice in a dual-channel supply chain, Mathematical Problems in Engineering, Vol. 2016, Article ID 4368326, doi: 10.1155/ $\underline{2016 / 4368326 .}$.

[22] Ha, A., Long, X., Nasiry, J. (2016). Quality in supply chain encroachment, Manufacturing \& Service Operations Management, Vol. 18, No. 2, 280-298, doi: $10.1287 / \mathrm{msom} .2015 .0562$.

[23] Jabarzare, N., Rasti-Barzoki, M. (2020). A game theoretic approach for pricing and determining quality level through coordination contracts in a dual-channel supply chain including manufacturer and packaging company, International Journal of Production Economics, Vol. 221, Article ID 107480, doi: 10.1016/i.ijpe.2019.09.001.

[24] Jafari, H., Hejazi, S.R., Rasti-Barzoki, M. (2016). Pricing decisions in dual-channel sup ply chain inclu ding mono polistic manufacturer and duopolistic retailers: A game-theoretic approach, Journal of Industry, Competition and Trade, Vol. 16, No. 3, 323-343, doi: 10.1007/s10842-016-0224-1.

[25] Zhou, J., Zhao, R., Wang, W. (2019). Pricing decision of a manufacturer in a dual-channel supply chain with asymmetric information, European Journal of Operational Research, Vol. 278, No. 3, 809-820, doi: 10.1016/i. ejor. 2019.05.006.

[26] Jian, M., Wang, Y.L. (2018). Decision-making strategies in supply chain management with a waste-averse and stockout-averse manufacturer, Advances in Production Engineering \& Management, Vol. 13, No. 3, 345-357, doi: 10.14743/apem2018.3.295. 


\title{
Usklajevanje dvokanalne dobavne verige s popolnimi izdelki glede na prodajni trud
}

\author{
Hu, H. ${ }^{\text {a, }}{ }^{*}$, Wu, Q. ${ }^{a}$, Han, S. ${ }^{a}$, Zhang, Z. ${ }^{a}$ \\ ${ }^{a}$ School of Economics and Management, Yanshan University, Qinghuangdao, P.R. China
}

\section{POVZETEK}

Ker vse več ljudi uporablja elektronsko poslovanje za nakupovanje, so proizvajalci pripravljeni odpreti spletne prodajne kanale, da bi ustvarili več dobička. Ta članek obravnava dvokanalno dobavno verigo (DCSC), ki jo sestavljata trgovec $\mathrm{s}$ tradicionalnim kanalom in proizvajalec $\mathrm{z}$ neposrednim kanalom. V zunanjem okolju negotovega tržnega povpraševanja in izdelkov proizvedenih z napakami, si proizvajalci prizadevajo za promocijo spletnih izdelkov, potrošniki pa se vedejo svobodno. Za analizo smo vzpostavili tri modele po teoriji iger pod vodstvom proizvajalca: a) nekooperativni model, b) model usklajevanja v okviru pogodbe o delitvi dohodka, c) model usklajevanja po pogodbi o delitvi dobička. Rezultati kažejo, da ima pogostost napake izdelka vpliv na določanje cen in truda za prodajo. Konkurenca med akterji dvojnega kanala je koristna za potrošnike, ki si prizadevajo za ustrezno ceno. Glede na celotni dobiček DCSC je sodelovanje med proizvajalcem in trgovcem bolj donosno kot konkurenca med njima, hkrati pa se proizvajalci in trgovci v primeru sodelovanja bolj trudijo za prodajo izdelkov. Pričakovani dobiček trgovca po pogodbi o delitvi dohodka je manjši od prihodka po pogodbi o delitvi dobička, vendar je skupni dobiček od modela usklajevanja večji od slednjega.

\section{PODATKIOČLANKU}

Ključne besede:

Elektronsko poslovanje;

Dobavna veriga;

Dvokanalna dobavna veriga

(DCSC);

Izdelek z napako;

Trud proizvajalca za prodajo;

Usklajevanje;

Teorija iger

*Kontaktna oseba: huhaiju@ysu.edu.cn (Hu, H.)

Zgodovina članka:

Prejet 22. aprila 2020

Popravljen 20. junija 2020

Sprejet 25. junija 2020 